\title{
Progress in Preparation and Application of Fabric Electrodes
}

\author{
Yunqi Zhai, Xiaoxia Liu, Ya Wang, Xiao Li \\ College of Fashion, Shanghai University of Engineering Science, Shanghai, China \\ Email: liuxiaoxialucky@126.com
}

Received 19 August 2014; revised 20 September 2014; accepted 17 November 2014

Academic Editor: Baozhong Sun, Donghua University, China

Copyright (C) 2014 by authors and Scientific Research Publishing Inc.

This work is licensed under the Creative Commons Attribution International License (CC BY). http://creativecommons.org/licenses/by/4.0/

(c) () Open Access

\begin{abstract}
Fabric electrode is an emerging electrode element; due to some of its advantages, it can replace traditional conductive gel to become the next generation of electrode. This paper starts from the definition of fabric electrode, introduces the definition and working principle of fabric electrode, materials of producing electrodes preparation methods, and highlights the fabric used in the myoelectric prosthetic electrodes, supercapacitors and ECG tests.
\end{abstract}

\section{Keywords}

Textile Electrode, ECG Monitoring, Myoelectric Prostheses, Super-Capacitor

\section{Introduction}

Electrode has an irreplaceable role in people's life. Conventional electrodes mainly include metal plate electrode, the attraction electrode, viscosity electrodes. Metal plate electrode is a metal plate mainly composed of nickel or copper silver, often used for limb lead electrodes, because the metal plate electrode has many drawbacks, such as poor anti-interference performance, prone to polarization, baseline drift and so on, it has been rarely used currently. Adsorption electrode is made of silver plated metal or nickel silver alloy, half spherical, back with porosity just likes a rubber ball, mainly used for chest electrode. However, when using the adsorbing electrode, the first step is squeezing the rubber ball to exclude the air in the ball, touch the electrodes to the skin, the skin irritation was not suitable for a long time monitoring. Sticky electrodes are usually made of $\mathrm{Ag} / \mathrm{AgCl}$, the electrode coated with conductive adhesive sticky. This method is also likely to cause some adverse human skin itching and other symptoms, and this can easily dehydrate conductive adhesive drying affect conductivity [1]. With the speed of the accelerated development of smart textiles, fabric electrode applications are increasingly common. 
Fabric electrode is a sensor that uses textile materials with textile structure development process of textile processing, and can sense the surface of human body bioelectricity. Currently, various structures of textile electrodes have been developed, including, woven, knitted, non-woven, embroidery, which are obtained by using conductive fibers produced or conventional fiber [2].

Fabric electrodes belong to dry electrode category, but because the substrate fabric electrodes are a variety of textile products, soft electrode surfaces can be bonded with the surface of the body, which reduces the artifact and noise generated by the motion between the electrode and the skin and inherits the advantages of the surface electrode. More favorable, the fabric electrode material is breathable, foldable, convenient and comfortable, washable, and can be applied on a long wearable medical device/on long wearable medical devices. In addition, it has got great attention of fabric electrodes in producing super capacitor due to its thin, flexible wearable use in power supply areas.

\section{Preparation of Fabric Electrodes}

The specific structure of the fabric is shown in Figure 1. The conductive cloth is wrapped with filler, the force after extrusion of which is to ensure conductive fabric fully contacted with human skin. The outside filler and the support pad electrode can be enhanced support force, and provide a buffer in the clothing worn by the external compression. Surface electrical conductive fabric can be passed through the wire to the signal input of the measurement unit ECG conditioning circuit [3].

\subsection{The Working Principle of Fabric Electrode}

According to the degree of polarization, electrode can also be divided into polarized electrode and non-polarizable electrode. Polarized electrode is applied current or voltage, contact electrode and electrolyte free of charge movement and displacement current; Non-polarizable electrode does not need to be a current or voltage, current automatically pass through the electrode and electrolyte interface. Because the fabric electrode is more consist of inert conductor, the inert material is very small solubility in the electrolyte. So when the electrode and electrolyte interface current is weak, the polarization potential of electrode is maintained in a certain constant value [4]. Fabric electrodes belong to inert non-polarized electrodes.

\subsection{Select of Fabric Electrode Material}

Ideal electrode materials could be divided into completely polarized materials and completely non-polarized material, and practical application electrode material is between them. Completely polarized material due to its electrochemical inert, could not be occurring reaction at higher stimulation; and not completely polarized material due to its low resistance, is suitable for weak signal measurement.

Currently, there are many metal materials used to make the fabric electrodes which are pure metal or wire, or metalized polymer filaments. Xu Pengjun [3] used key of silver nylon filament fiber as a basic fiber to do the fiber surface chlorination process, the composite structure is formed of silver chloride, silver-coated electrode produced fabric. Jang, S., et al. [5] used copper sputtering surface of the fabric to produce a conductive fabric treatment electrode. Wang, R.X. et al. [6] used a vacuum sputtering to do silver-plated nylon fabric surface treatment. Xu Jie, Li Meixia [7], who used the method of electroless nickel plating on the fabric substrate coated with a layer of metallic nickel and nickel-metal deposited on the surface of a conductive polymer having a catalytic

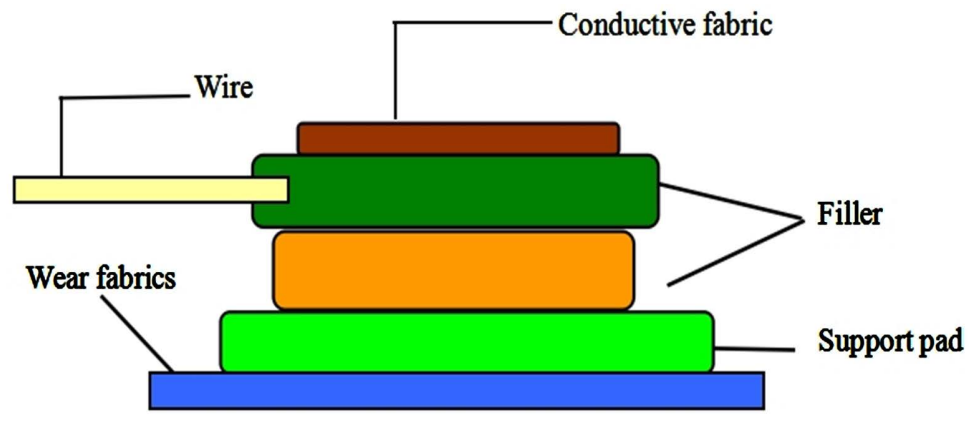

Figure 1. Structure of fabric electrode side. 
activity of making fabric electrodes. Yue Binbin, Ding Xin [8] got the gold-coated conductive fabric to make the fabric by magnetron sputtering electrode. Conductive fibers may also be used to obtain conventional fiber blend fabric electrodes. Ding Xin, Jin Lei, et al. [9], used medical grade with 76\% nylon and 24\% silver to plate elastic fiber blended fabrics made electrodes.

Besides the conductive yarns, the commonly used fabric electrode materials include conductive rubber and conductive inks. There are many kinds of rubber conductive material. Carbon-filled silicone rubber, nickelplated carbon-filled silicone rubber and silver-filled silicone rubber were made into fabric electrodes by printing, rapid prototyping, etc. [4] [9]. The benefits of using conductive rubber electrodes to produce fabric are: First, rubber biological applicability is good, and the surface is soft and could wear long-term which will not irritate the skin; Second, conductive rubber can be cleaned; Third, conductive rubber frivolous, flexibility, can be in the process of movement with the deformation of skin deformation and maximum block artifact produced by deformation signal and error. However, the defects in that the conductive rubber lie in, first, fabric electrode made from conductive rubber is not easy to connect the device; second, long time used may result in wear and the conductive metal outer layer decreased; third, the gel may leave residue on the skin, which can cause skin allergies and other adverse symptoms. Furthermore, there is the possibility of a short circuit when excess gel is applied in the electrode, another disadvantage is that the operating time is too short and the electrode of the singleuse. Furthermore, these processes are time-consuming preparation, uncomfortable, even painful to the patient, because the skin of the formulation generally comprises an outer skin abrasion. Repeated skin preparation and gel applications may also cause an allergic reaction or infection. In addition, ECG signal quality may degrade over an extended period of time as skin regeneration and/or conductive gel to dry.

Conductive ink, also known as silver paste ,printed on a substrate, which can make the substrate have a conductive property, usually printed on non-conductive material, such as plastic, glass, ceramic or cardboard, a conductive material is composed of silver and vaseline according to certain proportion, to increase conductivity, reduce the conductive contact resistance without fever [10]. The electrode surface produced by the conductive ink is smooth, long-term using and wearing will not stimulate the skin surface; Soft and flexible conductive ink electrode may be deformed with the deformation of the skin surface, but as long as the selection of substrates is right, it will not too soft to cause artifacts; Conductive ink is a silver metal compound in the polyester blend, which will not cause a degree of ground conductivity decreased [4]. Comparison of the three properties of electrode materials is shown in Table 1.

\section{Applications of Fabric Electrodes}

\subsection{Applications of Fabric Electrodes in the Myoelectric Prosthetic}

Traditional myoelectric prosthetic control signals are collected by the muscle surface $\mathrm{Ag} / \mathrm{AgCl}$ electrode gel metals, although the metal electrode conductive gel can provide high-quality EMG, but it is not convenient for clinical application of myoelectric prostheses [11]. As a new sensor, fabric electrode will be a new attempt multifunction myoelectric prosthetic control signal acquisition.

Zhang Zhiyong [12] used the conductive cloth copper plating fabrics to have been made of the electrode as shown in Figure 2. Electrode size was $2 \times 1.4 \mathrm{~cm}$, two electrodes were integrated together form a differential configuration. Textile electrodes mounted was made of rubber and nylon strap. Four able-bodied male subjects (without any nerve and muscle pathology) participated in the experimental study. Tests included eight arm and hand movements (Figure 3). The subjects were asked to watch a video presentation every movement, and synchronized with the video in the campaign, each action was repeated 10 times. For each repetition, subjects were asked to use the power of contraction of the most comfortable, and kept the contraction for about 5 seconds, then relaxed for 5 seconds.

\subsection{Applications of Fabric Electrodes in the Super Capacitor}

The super capacitor is also called double layer capacitor. Electric double layer capacitor is used the positive and negative ions in the electrolyte of the porous electrode to store energy, electric double layer forms electrolyte interface separation, which has a large capacity storage pulse discharge performance and excellent physical capacitors that do not have to performance, and far higher than the two battery power density and long service life. All the sensors, drives, and other electronic components on smart textiles need to have the power to provide. But the traditional power structures are rigid, the use of a wearable garment has great limitations. To solve the problem, 


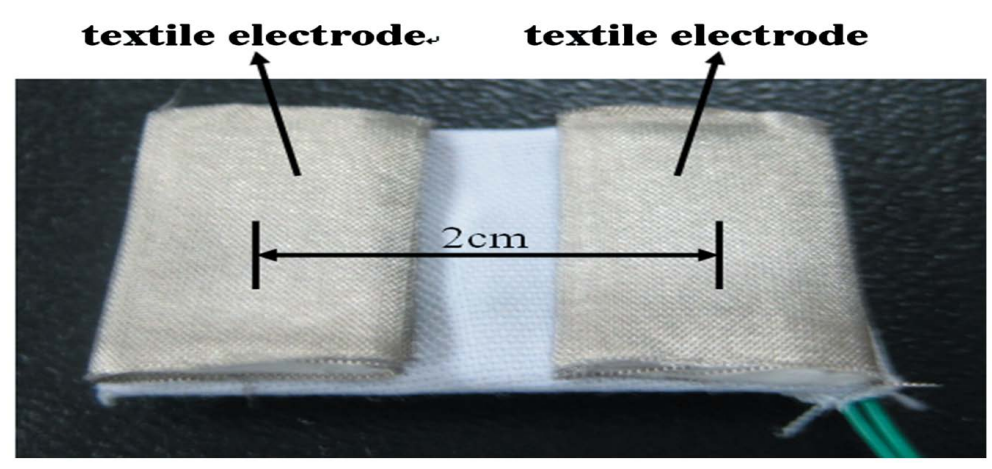

Figure 2. Fabric bipolar electrode.

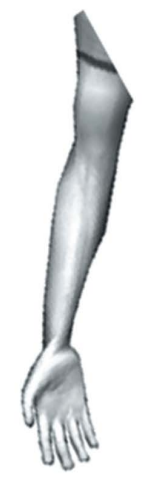

Elbow Extension
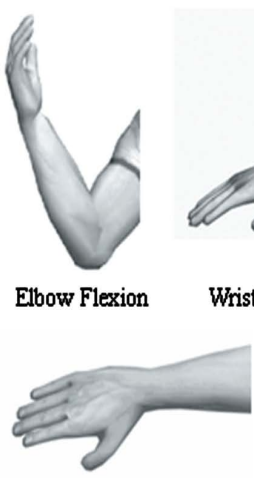

Wrist Extension

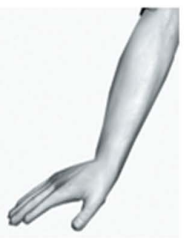

Wrist Extension

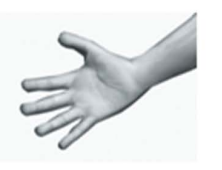

Hand Open
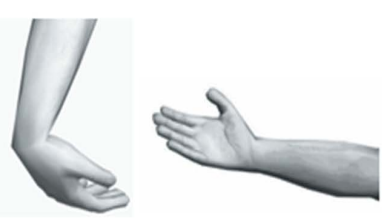

Wrist Supination

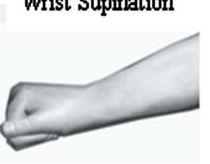

Hand Close

Figure 3. Study included eight arm and hand motions.

Table 1. Comparison of properties of three kinds of electrode materials.

\begin{tabular}{cccc}
\hline Performance & $\begin{array}{c}\text { Electrically conductive } \\
\text { thread }\end{array}$ & Conductive rubber & Conductive ink \\
\hline Biological applicability & Very good & Very good & Very good \\
Skin damage & Damage & Nothing & Nothing \\
Skin allergy & Nothing & Allergy & Nothing \\
Cleaning and repeatability & The general & Very good & Very good \\
After wearing the conductivity & Very good & Poor & Good \\
Connected with equipment & Simple & Complex & Goneral \\
The signal quality in sports & Poor & Very good & \\
\hline
\end{tabular}

it is necessary to develop a flexible, lightweight, portable storage device or energy conversion, and this means there must be a good tensile properties, that is, the performance does not occur in a stretched condition decay, and the ideal approach is that fabric structures forms such power. Super capacitor is an emerging energy storage device with high power density, and the charging time is short, excellent reversibility and long cycle life, energy conservation and so on.

Yue Binbin [13] used 90\% cotton and 10\% Lycra fibers to have made a stretchable knitted fabric, after the chemical reagent by a sputtering magnetron sputtering of gold metal on the fabric, and then in the presence of toluenesulfonic acid aqueous acetonitrile solution or pyrrole monomer to obtain poly pyrrole composite fabric by electrochemical polymerization method. By electrochemical experiments and tensile experiments, with high scalability and conductive fabric to give the pre-strain on the cotton fabric coated with gold could produce a simple electrochemical deposition. Poly pyrrole-coated fabric exhibited a surface resistance of $105 \Omega / \mathrm{m}$, and 
that after 1000 times stretching up to about $1645 \Omega / \mathrm{m}$ at 50\% stress. This indicated that the material exhibited good capacitance characteristics, even up to the scanning speed of $300 \mathrm{mV}, 10 \mathrm{mV}$ obtained at a scan rate of 255 capacitance. At $50 \%$ strain ratio increased slightly to 260 capacitors. Moreover, poly pyrrole coated cotton fabric had better cycling stability applying pressure, which may be attributed to the strain induced in the poly pyrrole be applied.

Comprehensive development of textile industry China Institute in Taiwan [14], which won the Orlando prize American 2011R \& D100 folding type fabric super capacitor was a lightweight and super capacitor completely. The capacitance, capacitance per unit mass was 10 times the general of the super capacitor. Combined with the research and development of non solvent based electrolyte, it not only solved the problem of environmental pollution, but also avoided the dangerous to use explosive. The super capacitor application has been locked in small power energy supply system and cell binding, including digital cameras, small wind turbines, electric bicycle and solar bags, such related products quality could greatly reduce the volume, and given the power function is more outstanding.

\subsection{Applications of Fabric Electrodes in the ECG Monitoring}

Wearable electrocardiogram detection is the clothes and ECG technology in people's daily wear combination, which can whenever and wherever possible in the natural state of the acquisition of ECG information of the human body. Due to introduce the wearable technology clothing and people usually wear clothes, so which is a safe and low physiology, low mental workload model detection technology [15]. Continuous ECG monitoring could be early detected of potentially dangerous human abnormal, and provide a safe living environment for patient. In addition, the dynamic ECG monitoring can ensure the normal practice for patients in daily life, rather than just receive medical care at home or in the hospital.

The fabric electrodes have replaced the traditional disposable Ag/AgCl electrodes, and solved the paste electrode leads to the patient skin allergy that could not be long-term used. At the same time, the fabric electrode ECG signal also used to collect the respiratory signal. Textile electrodes to detect ECG signals were usually integrated in a wearable system has a more complete description of the patient's health status.

Zheng Jiewen [16] used 10 pieces of fabric sewn on the inner electrode T-shirt with elastic, as shown in Figure 4. Intelligent T-shirt is made of elastic breathable cotton fabric, which could be made into different sizes and specifications. In order to adapt to the different shape and wear comfortable breathable, also the close contacting the T-shirts with elastic could keep the electrodes and the skin. This intelligent T-shirt electrode not only had the features of the ordinary cloth easily cutting and sewing, but also has been in accordance with the requirements of cutting to any shape, stitched and conductive property at any position inside of the T-shirt. Figure 2 and Figure 4

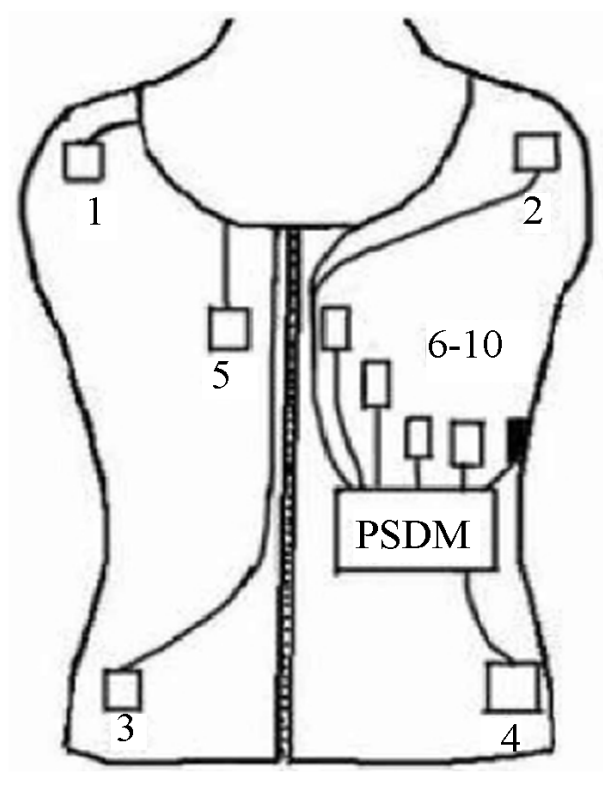

Figure 4. Intelligent T-shirt structure diagram. 
as ECG electrode at the same time, also used as the electrode impedance measurement of respiratory wave-form. The electrode was sutured to the fabric in the shirt, and the sponge placed inside the appropriate size, in order to increase the reliability of the electrode being contacting with skin. At the same time, with a thin layer of medical hydrogel in fabric electrode surface, the electrode could be contact with the skin well, and reduce the contacted impedance of skin, so as to not affect the wearable comfort conditions to obtain high quality ECG and respiratory signal.

\section{Conclusion}

With the improvement of people's living standard, it will be people's pursuit of being thin, light, easy to carry and multifunctional. The fabric electrode as an emerging electronic product, the patient's ECG monitoring and control of myoelectric prostheses gives people a new impression. In the age of pursuiting to be small, multifunctional, resistant to use, fabric electrodes, as a miniature of the super capacitor power is bound to get the attention of electronics manufacturers. Fabric electrode is soft, comfortable, breathable, and does not use conductive gel, the skin won't feel stimulated after using for a long time. Fabric electrode is easy to be used for wearable health monitoring in the clothing and it can be washed and reused; because of these characteristics, it has already got more and more attention from the researchers both at home and abroad. From its original use in hospital medical equipment to the use of the patient's ECG monitoring and electrical control of the prosthesis at home now, even the micro-power on the super capacitor in recent years, application of fabric electrode is expanding continuously to meet people's pursuit of some electronic products being small, thin and lightweight.

\section{References}

[1] Guo, W. (2012) Research and Implementation of Wearable Physiological Parameter Detection System. Jilin University, Changchun.

[2] Xu, P.J. (2012) Analysis and Dynamic Mechanical Action between Noise Study for the Textile Structure of the Electrode Surface ECG Monitoring with the Skin. Donghua University, Shanghai.

[3] Pereira, T., Carvalho, H., et al. (2013) Wearable Biopotential Measurement Using the TIADS1198 Analog Front-End and Textile Electrodes. IEEE Signal Conditioning and Signal Quality Assessment, 325-330.

[4] Tao, D.D. (2011) Research and EMG Prosthetic Fabric Electrode Signal Acquisition and Processing Technology. Harbin Institute of Technology, Harbin.

[5] Jang, S., et al. (2007) Exploring Possibilities of ECG Electrodes for Bio-Monitoring Smartwear with Cu Sputtered Fabrics. Human-Computer Interaction. Interaction Platforms and Techniques, 4551, 1130-1137. http://dx.doi.org/10.1007/978-3-540-73107-8_124

[6] Wang, R.X., et al. (2010) Microstructures and Electrical Conductance of Silver Nanocrystalline Thin Films on Flexible Polymer Substrates. Surface and Coatings Technology, 204, 1206-1210. http://dx.doi.org/10.1016/j.surfcoat.2009.10.030

[7] Xu, J. (2013) A Method for Preparing the Dye-Sensitized Solar Cell Fabric Electrode. Chinese Patent, 103258650.2013-08-21.

[8] Yue, B.B. and Ding, X. (2012) A Method for Preparing a Flexible Super Capacitor Electrode Material. Chinese Patent, 102779648.2012-11-14.

[9] Ding, X. and Jin, L. (2012) Based on the Non-Contact Portable Sleep Monitoring System Fabric ECG Electrode. Beijing Biomedical, 31, 293-297.

[10] Wang, T.T. (2011) Conductive Ink and RFID. Printing Quality \& Standardization, 11, 14-16.

[11] Zhang, Z.Y., Tao, D.D., et al. (2010) Performance Analysis of Textile Sensors in Control of Multifunctional Myoelectric Prostheses. Bulletin of Advanced Technology Research, 4, 1-6.

[12] Zhang, Z.Y. (2010) EMG Signal Acquisition and Myoelectric Prosthesis Control on. Harbin Institute of Technology, Harbin.

[13] Yue, B.B., Wang, C.Y., Ding, X., et al. (2013) Electrochemically Synthesized Stretchable Polypyrrole/Fabric Electrodes for Supercapacitor. Electrochimica Acta, 113, 17-22. http://dx.doi.org/10.1016/j.electacta.2013.09.024

[14] Liu, C.N. (2011) The Super Capacitor Dynamic. Power Technology, 35, 1485-1486.

[15] Ruffini, G., Dunne, S., Farres, E., et al. (2006) ENOBIO-First Tests of a Dry Electrophysiology Electrode Using Carbon Nanotubes. 28th Annual International Conference of the IEEE Engineering in Medicine and Biology Society, 
New York, 30 August-3 September 2006, 1826-1829.

[16] Zheng, J.W. (2008) Wearable Real-Time Diagnosis, Alarm, Mobile Health Monitoring System. Military Science and Medical School Students Equipment Research Institute, Beijing. 
Scientific Research Publishing (SCIRP) is one of the largest Open Access journal publishers. It is currently publishing more than 200 open access, online, peer-reviewed journals covering a wide range of academic disciplines. SCIRP serves the worldwide academic communities and contributes to the progress and application of science with its publication.

Other selected journals from SCIRP are listed as below. Submit your manuscript to us via either submit@scirp.org or Online Submission Portal.
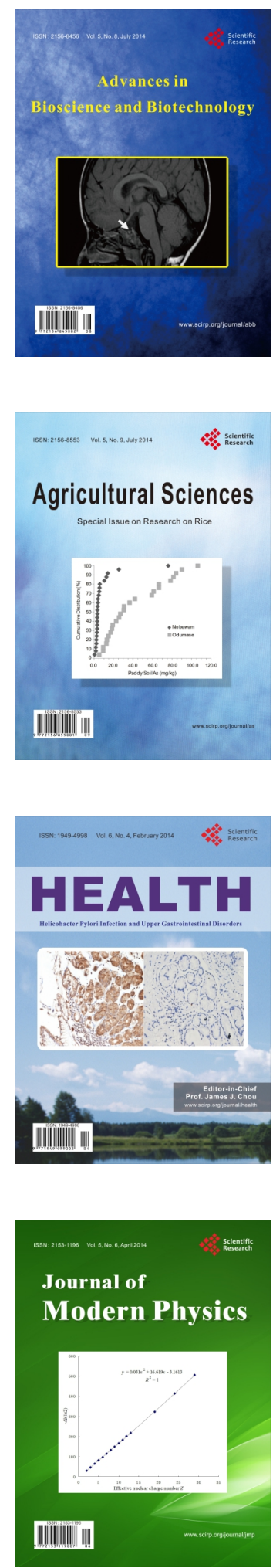
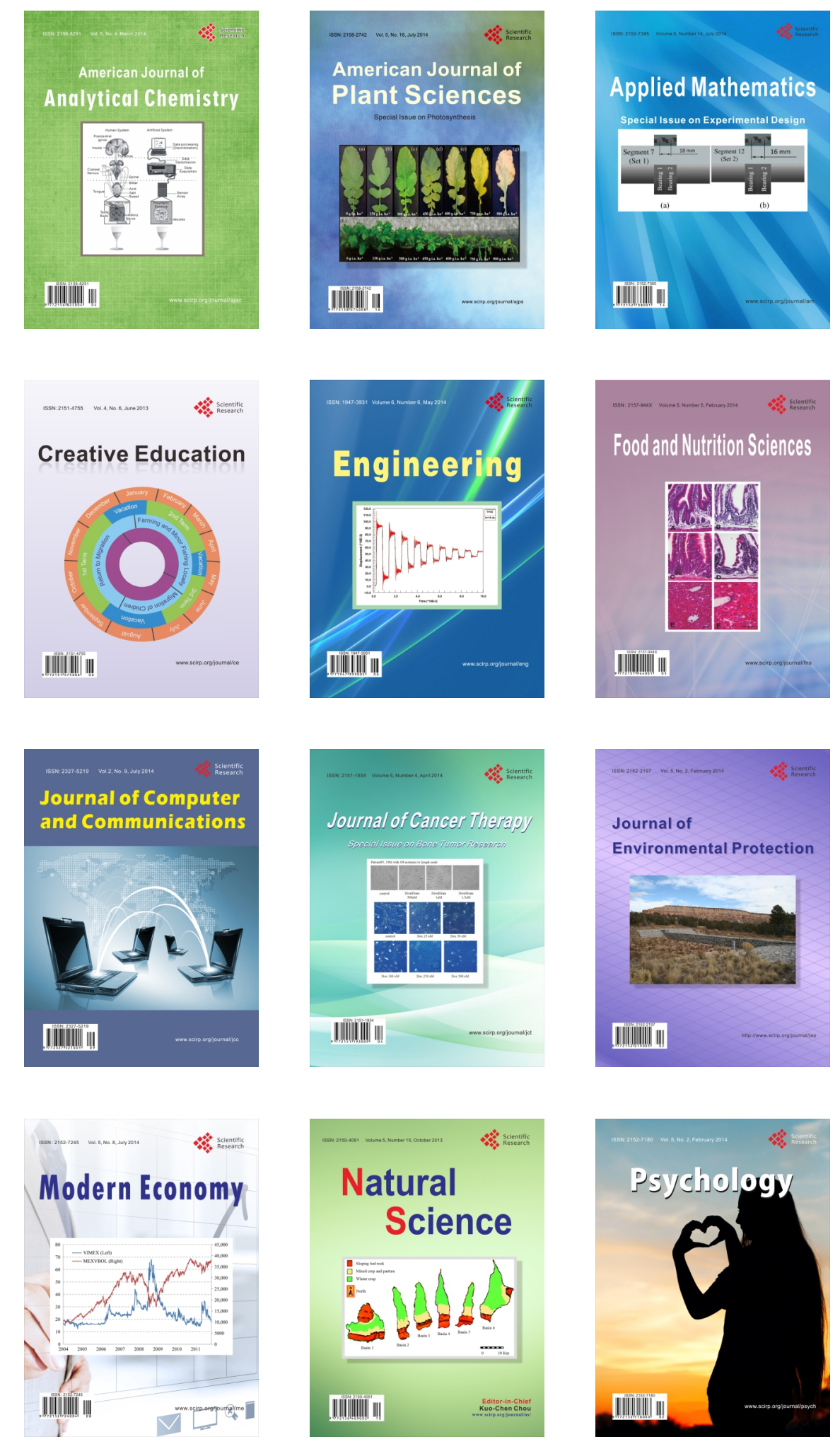